\title{
Preparation and Thermal Behavior of Fluorine-Containing Phenylmaleimides
}

\author{
Akira NagAI and Akio TAKaHASHI \\ Hitachi Research Laboratory, Hitachi Ltd., 7-1-1 Omika-cho, \\ Hitachi, Ibaraki 319-12, Japan
}

(Received September 3, 1993)

\begin{abstract}
Fluorine content dependency on the melting point and thermal polymerization reactivity of fluorine-containing phenylmaleimides were investigated by differential scanning calorimetry and are discussed on the basis of molecular structure calculations. The syntheses of fluorine-containing maleimides were carried out by thermal dehydration. Their yields tended to be lower with increasing fluorine content. This was due to lowered nucleophilic reactivity of the amino group. The fluorine-containing maleimides tended to have a high melting point with increasing fluorine content. But symmetry in the chemical structure was a more important factor influencing the melting point. The thermal polymerization temperature of double bonds became higher with increasing fluorine content. This thermal polymerization reactivity could be explained from results of molecular structure calculations. The decreased thermal polymerization reactivity of fluorine-containing maleimides could be attributed to molecular stabilization or the difficulty of activated biradical formation as shown by MNDO or MM method calculations. This is in agreement with that of fluorine-containing bismaleimides.

KEY WORDS Maleimide / Fluoro-Containing / Melting Point / Polymerization Reactivity / MNDO /
\end{abstract}

The maleimide structure has been utilized to produce thermally stable polymers. For example, bismaleimide resin provides a thermoset product which has been applied in a wide range of composite materials. ${ }^{1-3}$ The unsaturated double bonds of maleimide rings can thermally polymerize without the formation of volatile by-products which may cause voids. On this point, they differ from other condensation-type polyimides. Therefore, the thermal polymerization reactivity of double bonds of maleimide has been investigated and maleimide compounds have been widely used in the electronics industry. ${ }^{4-6}$ Among them, fluorinecontaining compounds have several good properties which are thought suited for electronics materials. These are a low dielectric constant, excellent thermal stability, and high flame retardancy. ${ }^{7}$ In general, a fluorine substituent might lower thermal polymeriza- tion reactivity of double bonds of maleimide due to its electron-withdrawing effect. ${ }^{8,9}$ Thus investigation of reactivity of fluorine-containing maleimides is of interest. The thermal properties, melting point, and polymerization temperature, of fluorine-containing bismaleimides have been investigated. ${ }^{7}$ These bismaleimides have flexible ether groups and a long phenoxy chain within a monomer structure. Therefore, the cured product has good mechanical properties such as high flexural strength and elongation. ${ }^{10}$ Moreover, the fluorine-containing compound has good electrical and thermal properties and is well suitable for electronics materials. It would be of interest to investigate the fluorine introducing dependency on these thermal properties. The fluorine-containing phenylmaleimide structure was selected as the model compound for the analysis of the fluorine content dependence. 
In this paper, fluorine-containing phenylmaleimides were synthesized and the fluorine content dependency on their melting point and thermal polymerization reactivity was measured by differential scanning calorimetry. The results are discussed on the basis of molecular structure calculations.

\section{EXPERIMENTAL}

Syntheses of Fluorine-Containing Phenylmaleimides

All chemicals were purchased from Wako Pure Chemical. The chemical structures of the maleimides used are shown in Figure 1. The phenylmaleimide (0FMI) was commercially

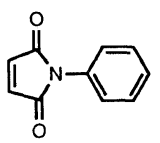

OFMI $\mathrm{mp}=88^{\circ} \mathrm{C}\left(\text { lit. } 89^{\circ} \mathrm{C}\right)^{\mathrm{a}}$

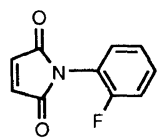

(lit. $\left.76^{\circ} \mathrm{C}\right)^{\mathrm{b}}$

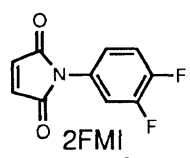

$\mathrm{mp}=114^{\circ} \mathrm{C}$

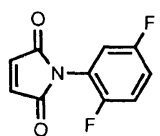

(lit. $\left.97^{\circ} \mathrm{C}\right)^{\mathrm{b}}$

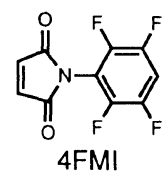

$\mathrm{mp}=139^{\circ} \mathrm{C}\left(\text { lit. } 137^{\circ} \mathrm{C}\right)^{\mathrm{b}}$

a: Merck Index 11th ed.

b: J.M.Barrales-Rienda, et al., J. Fluorine Chem. 1977, 9, 293

Figure 1. Chemical structures and melting points of phenylmaleimides. available and purified by recrystallization with acetone and $n$-hexane. In general, maleimides are prepared by chemical dehydration (cyclization) with acetic anhydride, following Searle's procedure. ${ }^{11}$ This method gives maleimides after a relatively short reaction time and at low reaction temperature. The fluorine-containing maleimides were also synthesized by the reaction of fluorine-containing phenylamines with maleic anhydride according to substantial modifications made to the reaction conditions used by Searle's method. ${ }^{12}$ However, this method had a low yield in the case of high fluorine content maleimides and purification of products was relatively difficult because of contamination from several catalysts such as tertiary amine or metal acetate salt. Therefore, in this study, fluorine-containing maleimides are synthesized from the corresponding amines and maleic anhydride by a one-step process. This process requires higher temperature and longer reaction time to get thermal dehydration as compared with Seale's method. ${ }^{13}$ Moreover, excess amount of maleic anhydride is used. This process, however, seems effective for amines which have amino groups of low reactivity and high fluorine content. ${ }^{14}$

The procedure was as follows. Amine $(0.05$ mol) in acetic acid solution $(50 \mathrm{ml})$ was added dropwise to maleic anhydride $(0.15 \mathrm{~mol})$ in acetic acid solution $(150 \mathrm{ml})$. During addition ( $30 \mathrm{~min})$, the mixture was stirred and nitrogen was bubbled in. The mixture was kept at room temperature $\left(c a .25^{\circ} \mathrm{C}\right)$. After all the amine had been added, stirring and nitrogen bubbling were continued for $30 \mathrm{~min}$. The mixture was refluxed at $120^{\circ} \mathrm{C}$, for $15 \mathrm{~h}$, under nitrogen. The product was re-precipitated three times with water and acetone. Then it was dissolved in acetone and re-crystallized with $n$-hexane. This crystalline product was filtered and dried in vacuo. The yields are listed in Table I. The five fluorine-containing phenylmaleimides shown in Figure 1 (1FMI, 2FMI, 3FMI, 4FMI, and 5FMI) could be obtained by the thermal dehydration process. The yields decreased with 
increasing fluorine content. This was attributed to lowered nucleophilic reactivity of the amino group.

\section{Apparatus and Calculations}

Differential scanning calorimetry measurements were made on a Du Pont 910 DSC Instrument at a heating rate of $10 \mathrm{~K} \mathrm{~min}^{-1}$ in air. The sample weight was about $2.5 \mathrm{mg}$.

Molecular structure calculations were carried out with the Hypercube Inc. Hyperchem program. Molecular mechanics (MM) and modified neglect of diatomic overlap (MNDO) methods were used. The MM method uses valence geometric optimization to estimate energetics from bond lengths and angle potentials of the constituent atoms. Stabilization of maleimide molecules was calculated by the MM method. MNDO is a several semi-empirical molecular orbital method and was used to obtain molecular orbital energies and electron densities. In this work, the AM1 (Austin Model 1) method, developed by Dewar was selected for the MNDO calculations because it is well-suited for heterocyclic structures such as the maleimides. ${ }^{15}$

\section{RESULTS AND DISCUSSION}

\section{Melting Point}

- The results obtained from DSC measurements are listed in Table I. Fluorine-containing phenylmaleimides have high melting points compared with phenylmaleimide (0FMI). In general, a high molecular weight compound has a high melting point because the cohesive energy between molecules in the crystal state is large. Also, maleimide which has a symmetrical structure such as 1FMI and 4FMI has a relatively high melting point. This is presumed due to the high packing structure because there are strong intermolecular interactions in crystalline state. By contrast, the asymmetrical maleimides, 2FMI and 3FMI, have lower melting points compared with 1FMI, although their molecular weights are higher. This is considered due to the low packing density of molecules in the crystalline state and possible existence of weak intermolecular interactions. Consequently, the molar heat of fusion $\left(\Delta H_{\mathrm{f}}\right)$ in asymmetrical molecules tends to be smaller than that of symmetrical molecules as shown in Table I.

Other phenylmaleimides with their reported melting points ${ }^{12}$ are shown in Figure 1 for comparison with the results of this study. For monofluorophenylmaleimides, asymmetrical compounds such as ortho and metha substituted maleimides have lower melting points compared with 1FMI and 0FMI, which have symmetrical structures. This suggests that symmetry in the molecular structure is an extremely important factor affecting melting point.

5FMI has a relatively low melting point although symmetrical structure and high

Table I. Fusion behavior of phenylmaleimides ${ }^{a}$

\begin{tabular}{|c|c|c|c|c|c|c|}
\hline \multirow{2}{*}{ Compound } & Yield & \multirow{2}{*}{ MW } & \multirow{2}{*}{$\frac{T_{\mathrm{m}}}{{ }^{\circ} \mathrm{C}}$} & \multirow{2}{*}{$\frac{\Delta h_{\mathrm{f}}}{\mathrm{Jg}^{-1}}$} & \multirow{2}{*}{$\frac{\Delta H_{\mathrm{f}}}{\mathrm{kJmol}^{-1}}$} & \multirow{2}{*}{$\frac{\Delta S_{\mathrm{f}}}{\mathrm{Jmol}^{-1} \mathrm{k}^{-1}}$} \\
\hline & $\%$ & & & & & \\
\hline 0FMI & & 173 & 88 & 127 & 22.0 & 60.9 \\
\hline 1FMI & 60.7 & 191 & 155 & 148 & 28.3 & 66.1 \\
\hline 2FMI & 57.7 & 209 & 114 & 113 & 23.6 & 61.0 \\
\hline 3FMI & 45.3 & 227 & 105 & 118 & 26.8 & 70.9 \\
\hline 4FMI & 49.0 & 245 & 139 & 135 & 33.1 & 80.3 \\
\hline 5FMI & 33.3 & 263 & 106 & 88 & 23.1 & 60.9 \\
\hline
\end{tabular}

a $\quad \mathrm{WW}$, molecular weight; $T_{\mathrm{m}}$, melting point; $\Delta h_{\mathrm{f}}$, specific heat of fusion; $\Delta H_{\mathrm{f}}$, molar heat of fusion; $\Delta S_{\mathrm{f}}$, molar entropy change of fusion. 
molecular weight. The reason for this is not clear yet. The molar heat and entropy change of fusion in 5FMI are also relatively low compared with other fluorine-containing phenylmaleimides. This is presumably due to difference molecular structure, for example, the different torsional angle between the phenyl and maleimide rings.

\section{Thermal Polymerization}

The thermal polymerization reactivity of fluorine-containing phenylmaleimides is expected to be reduced because fluorine groups have electron-withdrawing property. ${ }^{16}$ The initial polymerization temperatures are listed in Table II.

The initial polymerization temperature is higher with increasing fluorine content. This suggests that the reactivity is reduced by the introduction of fluorine substituents. The polymerization temperature could not be observed in the case of 1FMI. This is because 1FMI has a high melting point, which may be very close to the initial polymerization temperature. Therefore, polymerization might be concealed by the endothermic region of fusion. The electron density on the double carbon bonds was calculated by the MNDO

Table II. Polymerization temperature and molecular calculations

\begin{tabular}{cccccc}
\hline & \multicolumn{1}{c}{$T_{\mathrm{i}}$} & & $\Delta e$ & $E$ \\
\cline { 2 - 5 } \cline { 4 - 5 } & ${ }^{\circ} \mathrm{C}$ & & & $\mathrm{eV}$ & $\mathrm{eV}$ \\
\hline 0FMI & 163 & 0.158 & 7.67 & 1.52 \\
1FMI & $-^{\mathrm{a}}$ & 0.156 & 7.53 & 1.55 \\
2FMI & 171 & 0.155 & 7.59 & 1.65 \\
3FMI & 182 & 0.155 & 7.78 & 2.07 \\
4FMI & 234 & 0.155 & 8.14 & 2.57 \\
5FMI & 221 & 0.154 & 8.03 & 2.79 \\
\hline
\end{tabular}

a Polymerization temperature was not observed. $T_{\mathrm{i}}$, initial polymerization temperature; $\rho$, electron density on double bond; $\Delta e$, difference between LUMO and HOMO; LUMO, energy level of lowest unoccupied molecular orbital; HOMO, energy level of highest occupied molecular orbital; $E$, stabilization energy. method. The electron density becomes slightly lower with increasing fluorine content. However, this change does not seem to be so effective on change in the thermal polymerization reactivity. This result is in accord with earlier results from fluorine-containing bismaleimides. ${ }^{7}$

By contrast, the difference between HOMO and LUMO $(\Delta e)$ is strongly dependent on the fluorine content as shown in Table II. Here, HOMO represents the energy level of the highest occupied molecular orbital and LUMO is that of the lowest unoccupied one. This $\Delta \mathrm{e}$ is related to the ease of producing an activated biradical which is an excitation on intramolecular sites. The molecule is excited by the formation of a biradical structure produced when one electron transfers from HOMO to LUMO. Calculation of the excited biradical state should be done by an accurate configuration interaction method because the electron correlation is important for the excited state. But it is enormously time consuming and impractical. Therefore, in this study, LUMO of the excited state was approximated by the calculation of the ground state.

$\Delta e$ becomes higher on increasing fluorine content as stated above. With higher $\Delta e$, the thermal polymerization reactivity is reduced and formation of activated species does not occur easily. The relationship between initial polymerization temperature and $\Delta e$ is shown in Figure 2. A good correlation is obtained. It seems that the reactivity of the double bond can be estimated with $\Delta e$. Consequently, the activation energy of the thermal polymerization reaction is approximated by the bond dissociation energy of the double bond which represents the stabilization energy. As shown in Figure 2, this correlation is in also good accord with earlier results from bismaleimides. ${ }^{7}$

Thermal polymerization is treated as a reaction between the excited biradical species and the monomer as shown in Scheme 1. The excited biradical species has the SOMO and SOMO'. The maleimide monomer has HOMO 


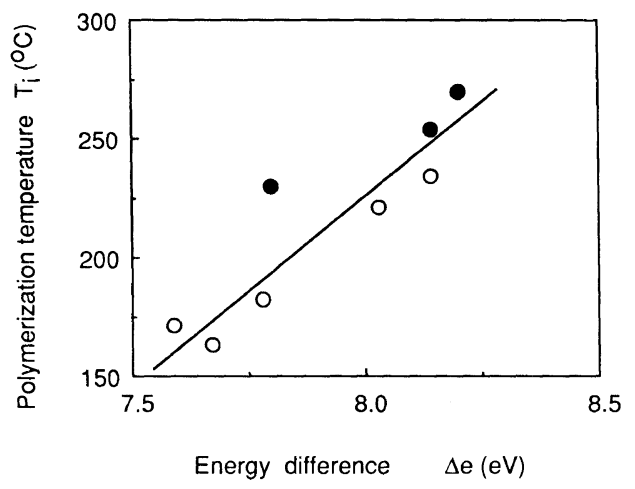

Figure 2. Relationship of initial polymerization temperature and energy difference in HOMO and LUMO. $\bigcirc$, phenylmaleimides in this work; $\mathbf{O}$, bismaleimides in ref 7 .

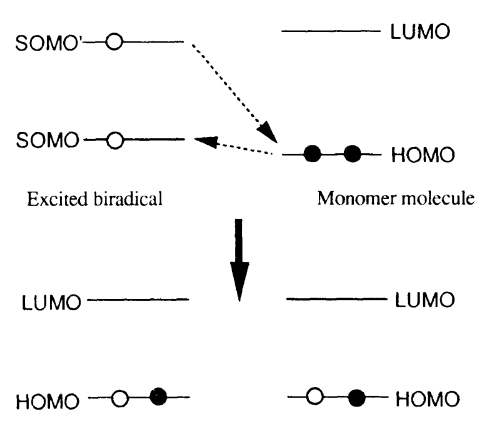

Scheme 1.

and LUMO. The energy level of SOMO is approximately that of HOMO and SOMO' is related to LUMO. As shown in Scheme 1, interactions of HOMO and SOMO, SOMO', and LUMO, respectively are important for the thermal polymerization reaction. As the energy levels of HOMO and SOMO are almost equal, the interaction is relatively strong. Therefore, the energy difference between SOMO' (this is almost equal to LUMO) and HOMO governs the thermal polymerization reactivity of maleimides. This suggests that the energy difference between HOMO and LUMO $(\Delta e)$ is closely related to the reactivity of thermal polymerization.

Stabilization of the molecule is also closely related to polymerization reactivity of the phenylmaleimides. The stabilization energy $(E)$ of the phenylmaleimides was calculated by

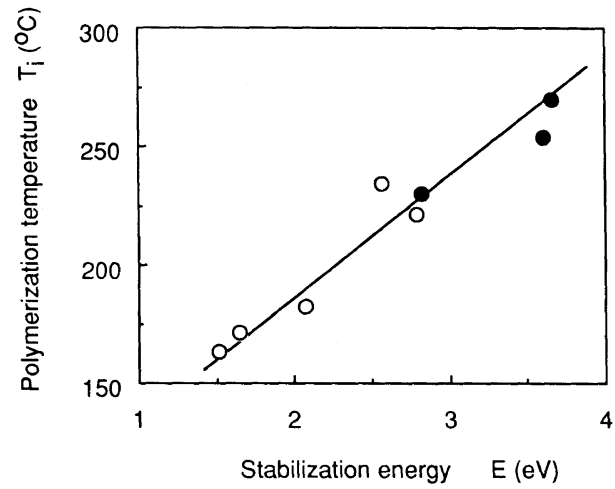

Figure 3. Relationship of initial polymerization temperature and molecular stabilization energy. $\bigcirc$, phenylmaleimides in this work, , bismaleimides in ref 7 .

the MM method and is listed in Table II. Phenylmaleimide compounds become more stable with increasing fluorine content. In general, molecular compounds are more stable with increasing molecular weight. Delocalization of $\pi$ electrons may also contribute to molecular stabilization with increasing fluorine content because fluorine has $\pi$ electrons on its $2 p$ orbitals and some of these electrons may interact with $\pi$ electrons on the phenyl and maleimide rings. The relationship between initial polymerization temperature and molecular stabilization energy is linear as show in Figure 3. The reactivities of bismaleimides fit well on the line. ${ }^{7}$ The fluorine-containing maleimides have low reactivity so they have a large energy gap between the excited and stable molecular structures and are not activated easily.

In conclusion, it was found that the thermal polymerization reactivity of maleimide compounds can be explained using ease of an activated biradical formation or molecular stabilization energy. The electron density on the carbon double bonds was not much affected by the electro-withdrawing characteristic of fluorine.

\section{REFERENCES}

1. T. Kataoka, Plastics, 33 (8), 70 (1982). 
2. A. Takahashi, S. Yokozawa, M. Wajima, and K. Tsukanishi, "Proceedings of Printed Circuit World Convention III", The Institute for Interconnecting and Packaging Electronic Circuits (IPC), Evanston, IL, U.S.A., 1984, W.C. III-14.

3. A. Takahashi, N. Ooki, A. Nagai, H. Akahoshi, A. Mukoh, and M. Wajima, IEEE Trans. CHMT, 15, 418 (1992).

4. J. V. Crivello, J. Polym. Sci. Polym. Chem. Ed., 11, 1185 (1973).

5. C. D. Giulio, M. Gautier, and B. Jasse, J. Appl. Polym. Sci., 29, 1771 (1984).

6. A. Nagai, A. Takahashi, M. Wajima, and K. Tsukanishi, Polym. J., 20, 125 (1988).

7. A. Nagai, A. Takahashi, M. Suzuki, and A. Mukoh, J. Appl. Polym. Sci., 44, 159 (1992).

8. S. Nishimura, A. Nagai, A. Takahashi, T. Narita, T. Hagiwara, and H. Hamana, Polym. J., 22, 171 (1990).
9. A. C. Mirsa, G.C. Tesoro, G. Hougham, and S. Pendharker, Polymer, 33, 1078 (1992).

10. A. Nagai, A. Takahashi, M. Suzuki, J. Katagiri, and A. Mukoh, J. Appl. Polym. Sci. 41, 2241 (1990).

11. N. E. Searle, US patent, 2444536 (1948).

12. J. M. Barrales-Rienda, J. G. Ramos, and M. S. Chaves, J. Fluorine Chem., 9, 293 (1977).

13. Ya. S. Vygodskii, V. A. Adigezalov, A. A. Askadskii, G. L. Slonimskii, Sh. T. Bagirov, V. V. Korshak, S. V. Vinogradova, and Z. M. Nagiev, Polym. Sci. U.S.S.R., 21, 2951 (1980).

14. A. C. Misra and G. Tesoro, Polymer, 33, 1083 (1992).

15. M. J. S. Dewar, E. G. Zoebisch, E. F. Healy, J. J. P. Stewart, J. Am. Chem. Soc. 107, 3902 (1985).

16. A. Matsumoto, T. Kubota, and T. Otsu, Macromolecules 23, 4508 (1990). 